\title{
Space Adaptive Finite Element Methods for Dynamic Signorini Problems
}

\author{
Heribert Blum · Andreas Rademacher · Andreas Schröder
}

the date of receipt and acceptance should be inserted later

\begin{abstract}
Space adaptive techniques for dynamic Signorini problems are discussed. For discretisation, the Newmark method in time and low order finite elements in space are used. For the global discretisation error in space, an a posteriori error estimate is derived on the basis of the semidiscrete problem in mixed form. This approach relies on an auxiliary problem, which takes the form of a variational equation. An adaptive method based on the estimate is applied to improve the finite element approximation. Numerical results illustrate the performance of the presented method.
\end{abstract}

Keywords Dynamic Signorini problem · A posteriori error estimation · Mesh refinement · Finite element method

\section{Introduction}

Dynamic Signorini problems arise in many engineering processes, e.g., in milling and grinding processes, vehicle design, and ballistics. In these processes, the main effects result from the contact at the surface of the bodies under consideration. Typical examples for engineering processes, where contact problems play a dominant role, are grinding processes. The workpiece interacts with the grinding wheel only in a small contact zone. However, the behaviour of the grinding machine is strongly affected by the resulting contact

Heribert Blum

Institute of Applied Mathematics, Technische Universität Dortmund, D-44221 Dortmund, Germany

E-mail: heribert.blum@mathematik.tu-dortmund.de

Andreas Rademacher

Institute of Applied Mathematics, Technische Universität Dortmund, D-44221 Dortmund, Germany

E-mail: andreas.rademacher@mathematik.tu-dortmund.de

Andreas Schröder

Department of Mathematics, Humboldt-Universität zu Berlin, Unter den Linden 6, D-10099 Berlin, Germany

E-mail: andreas.schroeder@mathematik.hu-berlin.de forces. A detailed study of this engineering problem is found in [1]. For the reliable simulation of such a process, a precise prediction is required for the contact forces, the contact zone and their effects onto the whole body. Furthermore, the contact zone and the contact forces are strongly depending on time. Hence, the precise consideration of these dependences is essential in the numerical simulation.

An adequate technique, which gives rise to a flexible and efficient finite element discretisation, is based on a posteriori error control and resulting adaptive mesh refinement. In general, a posteriori error estimates for second order hyperbolic problems are possible for two different discretisation approaches. One of them uses space time Galerkin methods for discretisation and applies similar techniques for error control as in the static case ([2-5]). The other one is based on finite differences in time and finite elements in space. Here, separate error estimators are used for the space and time direction ([6-8]) or error estimates for the whole problem ([9, 10]) are derived.

In this article, finite differences in time and finite elements in space are used to discretise the dynamic Signorini problems. Because only the data of the current time step comes into play, the error estimator can be evaluated efficiently. However, the separation of the space and time direction complicates the consideration of space time effects. The aim of this article is to derive an error estimator for the finite element discretisation in space direction. Therefore, an error control technique for static contact problems is applied to the semi discrete spatial problem. This technique goes back to Braess [11] and Schröder [12]. Other approaches to a posteriori error control for static contact problems are discussed in [1320]. In particular, an adaptive scheme for two-body contact is contained in [21]. Convergence results for adaptive algorithms in the context of obstacle problems are proven in [22]. 
Adaptivity in time direction is not taken into account in this article for notational simplicity, although it is easy to incorporate. One can do this on the basis of error estimators, which are known from the literature of second order hyperbolic problems [6].

The temporal discretisation of dynamic contact problems is a difficult task. Several approaches based on different problem formulations have been presented in literature. In [23] the penalty-method is used to solve the discrete problems. Special contact elements in combination with Lagrange multipliers are presented in [24]. Other techniques for smoothing and stabilizing the computation with special finite elements, e.g., Mortar finite elements, are presented in [2527]. In [28], the Newmark scheme is used with an additional $L^{2}$-projection for stabilization. Algorithms for dynamic contact/impact problems based on the energy- and momentum conservation are derived in $[29,30]$. An additive splitting of the acceleration into two parts, representing the interior forces and the contact forces, is the basis of the methods introduced in [31,32]. In [33-35] algorithms based on variational inequalities and optimisation algorithms are presented. Detailed surveys of this topic can be found in the monographs [36,37].

The article is organised as follows: In the next two sections, the strong and weak formulations of the dynamic simplified Signorini problem are introduced and the discretisation of the problem is discussed. In Section 4, the spatial error estimator is derived serving as the basis of an adaptive algorithm,which is explained in Section 5. In Section 6, two examples illustrate the application of the presented techniques. The article concludes with a discussion of the results.

\section{Continuous Formulation}

In this section, the strong and the weak formulation of the dynamic simplified Signorini problem are presented. Let $\Omega \subset$ $\mathbb{R}^{2}$ be the basic domain and $I:=[0, T] \subset \mathbb{R}$ a time interval. The boundary $\partial \Omega$ of $\Omega$ is divided into three mutually disjoint parts $\Gamma_{D}, \Gamma_{C}$ and $\Gamma_{N}$ with positive measure. Homogeneous Dirichlet and Neumann boundary conditions are prescribed on the closed set $\Gamma_{D}$ and on $\Gamma_{N}$, respectively. Contact may take place on the sufficiently smooth set $\Gamma_{C}, \bar{\Gamma}_{C} \subset \complement \Gamma_{D}$. See, e.g., [38], Section 5.3 for more details. The time dependent rigid foundation is parameterised by a sufficiently smooth function $g: \Gamma_{C} \times I \rightarrow \mathbb{R} \cup\{-\infty\}$. Here, the restriction $u \geq g$ on $\Gamma_{C}$ is considered, $u \leq g$ can be treated analogously.

The initial displacement $u_{0}$ is in

$H^{1}\left(\Omega, \Gamma_{D}\right):=\left\{v \in H^{1}(\Omega) \mid \gamma_{\Gamma_{D}}(v)=0\right\}$ and the initial velocity $v_{0}$ is in $L^{2}(\Omega)$. Here, $\gamma$ denotes the trace operator of functions in $H^{1}\left(\Omega, \Gamma_{D}\right)$ onto the boundary $\partial \Omega$. See, e.g., [39] for more details. The gradient of the displacement $u$ in space direction is denoted by $\nabla u$ and $\Delta u$ is the usual Laplace operator applied to $u$. The first and second time derivatives are denoted by $\dot{u}$ and $\ddot{u}$, respectively. In the following, all relations have to be understood almost everywhere.

We choose the unconstrained trial space

$V:=W^{2, \infty}\left(I ; L^{2}(\Omega)\right) \cap L^{\infty}\left(I ; H^{1}\left(\Omega, \Gamma_{D}\right)\right)$

for notational convenience, although the existence of a solution in $V$ can not be proven, even in the contact free case [39]. The set of admissible displacements is

$K:=\left\{\varphi \in V \mid \gamma_{\mid \Gamma_{C}}(\varphi) \geq g\right.$ on $\left.\Gamma_{C} \times I\right\}$.

The $L^{2}$-scalar product is defined by $(u, v)=\int_{\Omega} u v d x$ for $u, v \in L^{2}(\Omega)$. The density is set equal to 1 for notational simplicity. Eventually, the weak formulation of the simplified dynamic Signorini problem, see, e.g., [40], reads

Problem 2.1 Find a function $u \in K$ with $u(t=0)=u_{0}$ and $\dot{u}(t=0)=v_{0}$ for which

$$
\geq \begin{aligned}
(\ddot{u}(t), \varphi(t)-u(t))+(\nabla u(t), \nabla(\varphi(t)-u(t))) \\
\quad(f(t), \varphi(t)-u(t))
\end{aligned}
$$

holds for all $\varphi \in K$ and all $t \in I$.

Throughout this article, we assume $f \in L^{\infty}\left(I ; L^{2}(\Omega)\right)$.

If the solution is sufficiently smooth, we obtain the equivalent strong formulation (see [40])

$$
\begin{aligned}
\ddot{u}-\Delta u & =f & & \text { in } \Omega \times I \\
u & =0 & & \text { on } \Gamma_{D} \times I \\
\frac{\partial u}{\partial v_{d}} & =0 & & \text { on } \Gamma_{N} \times I \\
u-g & \geq 0 & & \text { on } \Gamma_{C} \times I \\
\frac{\partial u}{\partial v_{d}} & \leq 0 & & \text { on } \Gamma_{C} \times I \\
\frac{\partial u}{\partial v_{d}}(u-g) & =0 & & \text { on } \Gamma_{C} \times I,
\end{aligned}
$$

where $v_{d}$ is the outward normal direction on the boundary.

\section{Discretisation}

We use Rothe's method to discretise the dynamic simplified Signorini problem. First, the problem is discretised in temporal direction by the Newmark method (see [41]). The resulting spatial problems are approximately solved by low order finite elements. 


\subsection{Temporal Discretisation}

The time interval $I$ is split into $N$ equidistant subintervals $I_{n}:=\left(t_{n-1}, t_{n}\right]$ of length $k=t_{n}-t_{n-1}$ with $0=: t_{0}<t_{1}<$ $\ldots<t_{N-1}<t_{N}:=T$. The value of a function $w$ at a time instance $t_{n}$ is approximated by $w^{n}$. We use the notation $v=\dot{u}$ and $a=\ddot{u}$ for the velocity and the acceleration, respectively.

In the Newmark method, $v$ and $a$ are approximated by

$$
\begin{aligned}
a^{n} & =\frac{1}{\beta k^{2}}\left(u^{n}-u^{n-1}\right)-\frac{1}{\beta k} v^{n-1}-\left(\frac{1}{2 \beta}-1\right) a^{n-1}, \\
v^{n} & =v^{n-1}+k\left[(1-\alpha) a^{n-1}+\alpha a^{n}\right] .
\end{aligned}
$$

Here, $\alpha$ and $\beta$ are free parameters in the interval $[0,2]$. For second order convergence, $\alpha=\frac{1}{2}$ is required. Furthermore, the inequality $2 \beta \geq \alpha \geq \frac{1}{2}$ has to be valid for unconditional stability (see [42]). For dynamic contact problems, the choice $\alpha=\beta=\frac{1}{2}$ is recommended to guarantee conservation of energy and momentum (see [24,35]). For starting the Newmark method the initial acceleration $a_{0}$ is needed. It can be calculated on the basis of the initial displacement by solving the equation

$$
\left(a^{0}, \varphi\right)+\left(\nabla u^{0}, \nabla \varphi\right)=(f(0), \varphi) .
$$

Here, we assume that no contact takes place in the initial configuration (see [42] for more details). The semi-discrete problem then reads as follows:

Algorithm 3.1 Find $u$ with $u^{0}=u_{0}, v^{0}=v_{0}$ and $a^{0}=a_{0}$, such that in every time step $n \in\{1,2, \ldots, N\}$, the function $u^{n} \in K^{n}$ is the solution of the variational inequality

$\left(a^{n}, \varphi-u^{n}\right)+\left(\nabla u^{n}, \nabla\left(\varphi-u^{n}\right)\right) \geq\left(f\left(t_{n}\right), \varphi-u^{n}\right)$,

for all $\varphi \in K^{n}$. The quantities $u^{n}, v^{n}$, and $a^{n}$ are coupled by the equations (3.1) and (3.2).

The set $K^{n}:=\left\{\varphi \in H^{1}\left(\Omega, \Gamma_{D}\right) \mid \gamma_{\Gamma_{C}}(\varphi) \geq g^{n}\right.$ on $\left.\Omega\right\}$ is the time discretized set of the admissible displacements. Substituting the equation (3.1) with $\alpha=\beta=\frac{1}{2}$ in the inequality (3.3) leads to

$$
\begin{aligned}
& \left(u^{n}, \varphi-u^{n}\right)+\frac{1}{2} k^{2}\left(\nabla u^{n}, \nabla\left(\varphi-u^{n}\right)\right) \\
\geq & \left(\frac{1}{2} k^{2} f\left(t_{n}\right)+u^{n-1}+k v^{n-1}, \varphi-u^{n}\right) .
\end{aligned}
$$

This can be written as

$c\left(u^{n}, \varphi-u^{n}\right) \geq\left(F^{n}, \varphi-u^{n}\right)$,

where $c$ is defined by

$c(\omega, \varphi):=(\omega, \varphi)+\frac{1}{2} k^{2}(\nabla \omega, \nabla \varphi)$

and $F^{n}$ as

$F^{n}:=\frac{1}{2} k^{2} f\left(t_{n}\right)+u^{n-1}+k v^{n-1}$.
The bilinear form $c$ is uniformly elliptic, continuous, and symmetric. Thus, an elliptic variational inequality has to be solved in each time step. An efficient way for solving variational inequalities is given by their mixed formulation. The Lagrange parameters may be interpreted as contact forces. The variational inequality (3.4) is equivalent to the following mixed problem:

Algorithm 3.2 Find $(u, \lambda)$ with $u^{0}=u_{0}, v^{0}=v_{0}$, and $a^{0}=$ $a_{0}$, such that $\left(u^{n}, \lambda^{n}\right) \in V_{k} \times \Lambda_{k}$ is the solution of the system

$$
\begin{gathered}
c\left(u^{n}, \varphi\right)+\left\langle\lambda^{n}, \gamma_{\mid \Gamma_{C}}(\varphi)\right\rangle=\left(F^{n}, \varphi\right) \\
\left\langle\mu-\lambda^{n}, \gamma_{\mid \Gamma_{C}}\left(u^{n}\right)-g^{n}\right\rangle \leq 0,
\end{gathered}
$$

for all $\varphi \in V_{k}$, all $\mu \in \Lambda_{k}$ and all $n \in\{1,2, \ldots, N\}$. Based on the equations (3.1) and (3.2), the functions $v^{n}$ and $a^{n}$ are calculated in a postprocessing step.

Here, $\Lambda_{k}$ is the dual cone of the set

$G:=\left\{\mu \in H^{1 / 2}\left(\Gamma_{C}\right) \mid \mu \leq 0\right\}$.

The dual pairing is expressed by $\langle\cdot, \cdot\rangle$. The set $V_{k}:=H^{1}\left(\Omega, \Gamma_{D}\right)$ is the unconstrained trial space discretised in time. The equivalence of the two formulations is a well-known conclusion from the general theory of minimisation problems in Hilbert spaces presented, e.g., in $[44,45]$.

\subsection{Spatial Discretisation}

A finite element approach is applied to discretise the Algorithm 3.2 based on the mixed formulation. We use adaptive algorithms with dynamic meshes. Therefore, the trial spaces $V_{h}^{n}$ and $\Lambda_{H}^{n}$ may vary from time step to time step. Bilinear basis functions on the mesh $\mathbb{T}^{n}$ are used for the finite element space $V_{h}^{n}$. The discrete Lagrange multipliers are piecewise constant and are contained in the set $\Lambda_{H}^{n}$. The index $H$ indicates that coarser meshes may be chosen for the Lagrange multipliers. In our calculations, we use $H=2 h$ for stability reasons. In Figure 3.1, the results for the Lagrange multiplier with different $H$ are compared. The solution for $H=h$ is obviously not stable, whereas stability is observed for $H=2 h$.

Because of the varying meshes, FE-functions have to be transfered to the mesh of the current time step. This process is denoted by $I_{h}$ and is realized by an $L^{2}$-projection. One might also consider standard interpolation as a transfer operator, which needs less effort, but can lead to instabilities. The discrete problem in space and time is

Algorithm 3.3 Find $\left(u_{h}^{n}, \lambda_{H}^{n}\right) \in V_{h}^{n} \times \Lambda_{H}^{n}$ with $u_{h}^{0}=I_{h} u_{0}$, $v_{h}^{0}=I_{h} v_{0}$ and $a_{h}^{0}=I_{h} a_{0}$, such that the system

$c\left(u_{h}^{n}, \varphi_{h}\right)+\left\langle\lambda_{H}^{n}, \gamma_{\mid \Gamma_{C}}\left(\varphi_{h}\right)\right\rangle=\left(F_{h}^{n}, \varphi_{h}\right)$

$$
\left\langle\mu_{H}-\lambda_{H}^{n}, \gamma_{\mid \Gamma_{C}}\left(u_{h}^{n}\right)-g^{n}\right\rangle \leq 0
$$




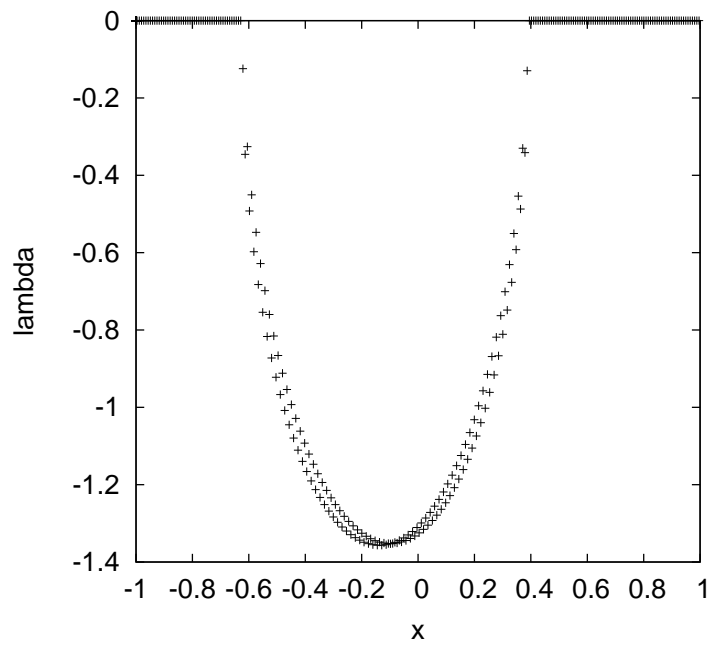

(a) Lagrange multiplier with $H=h$

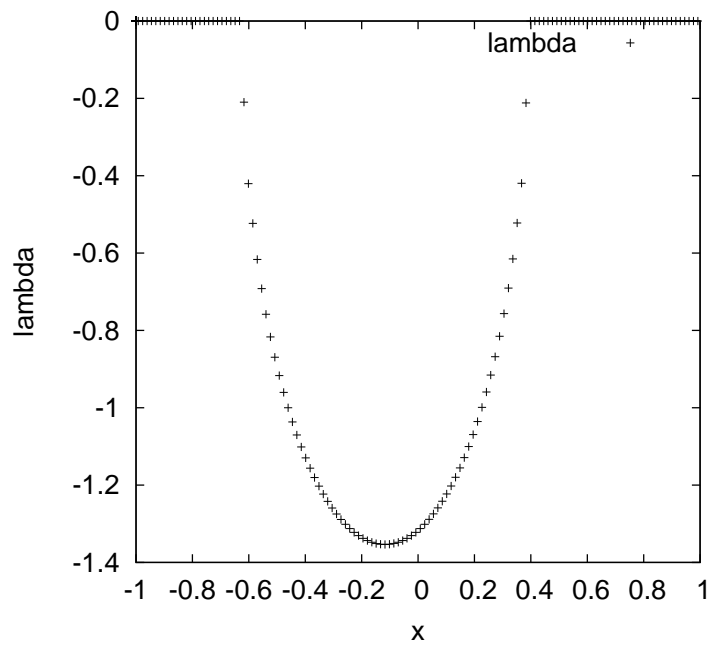

(b) Lagrange multiplier with $H=2 h$

Fig. 3.1 Results for the Lagrange multiplier with different $H$.

is valid for all $\varphi_{h} \in V_{h}^{n}$ and $\mu_{H} \in \Lambda_{H}^{n}, n \in\{1,2, \ldots, N\}$. Additionally, the equations (3.1) and (3.2) determine $v_{h}^{n}$ and $a_{h}^{n}$.

Here, $F_{h}^{n}$ is given by

$F_{h}^{n}:=\frac{1}{2} k^{2} f\left(t_{n}\right)+I_{h} u_{h}^{n-1}+k I_{h} v_{h}^{n-1}$.

The system (3.7-3.8) leads to the following saddle point problem in $\mathbb{R}^{m}$, where $m$ depends on the time level $n$ :

$$
\begin{aligned}
A^{n} \bar{u}^{n}+B^{n} \bar{\lambda}^{n} & =\bar{F}^{n} \\
\left(\bar{\mu}-\bar{\lambda}^{n}\right)^{T}\left(\left(B^{n}\right)^{T} \bar{u}^{n}-\bar{g}^{n}\right) & \leq 0,
\end{aligned}
$$

which must hold for all $\bar{\mu} \in \mathbb{R}_{\leq 0}^{\tilde{m}}$. Here, $A^{n}:=M^{n}+\frac{1}{2} k^{2} K^{n}$ is the generalised stiffness matrix, $M^{n} \in \mathbb{R}^{m \times m}$ is the mass matrix and $K^{n} \in \mathbb{R}^{m \times m}$ is the stiffness matrix. The matrix
$B^{n} \in \mathbb{R}^{m \times \tilde{m}}$ represents the dual pairing in (3.8). Notice, that all matrices may change from time step to time step.

Using the Schur complement $\bar{u}^{n}:=\left(A^{n}\right)^{-1}\left(\bar{F}^{n}-B^{n} \bar{\lambda}^{n}\right)$, the saddle point problem is rewritten as the quadratic program

$$
\begin{array}{cc}
\min _{\bar{\lambda}^{n} \in \mathbb{R}^{\tilde{m}}} & \frac{1}{2}\left(\bar{\lambda}^{n}\right)^{T} Q^{n} \bar{\lambda}^{n}-\left(\bar{\lambda}^{n}\right)^{T}\left[\left(B^{n}\right)^{T}\left(A^{n}\right)^{-1} \bar{F}-\bar{g}^{n}\right] \\
\text { s. t. } & \bar{\lambda}^{n} \leq 0 .
\end{array}
$$

The matrix $Q^{n}:=\left(B^{n}\right)^{T}\left(A^{n}\right)^{-1} B^{n}$ is symmetric and positive semidefinite. Thus, a standard quadratic program with simple sign constraints has to be solved in each time step. For the numerical solution of this quadratic program (QP) any QP-solver can be used, which only requires a user-defined routine for the calculation of $Q^{n} \bar{\lambda}^{n}$.

\section{Spatial Error Estimation}

In this section, an error estimation is derived for the spatial error in every time step. The estimation is easy to implement and can be evaluated fast. The temporal error is not considered. The idea of the error estimation goes back to Braess [11], who presented it for static obstacle problems. This idea was extended by Schröder [12] to static Signorini problems even with friction by introducing a general framework for error control of variational inequalities in Hilbert spaces. Here, we extend this approach to time-dependent Signorini problems by applying the general framework to the semi-discrete Algorithm 3.1. Since the function $F^{n}$ is unknown in practice, we have to substitute $F^{n}$ by $F_{h}^{n}$ in Algorithm 3.1 to obtain an error estimator, which can be evaluated. This leads to the following saddle point problem:

Auxiliary problem 4.1 Find $\left(\tilde{u}^{n}, \tilde{\lambda}^{n}\right) \in V_{k} \times \Lambda_{k}$, such that

$$
\begin{gathered}
c\left(\tilde{u}^{n}, \varphi\right)+\left\langle\tilde{\lambda}^{n}, \gamma_{\mid \Gamma_{C}}(\varphi)\right\rangle=\left(F_{h}^{n}, \varphi\right) \\
\left\langle\mu-\tilde{\lambda}^{n}, \gamma_{\mid \Gamma_{C}}\left(\tilde{u}^{n}\right)-g^{n}\right\rangle \leq 0
\end{gathered}
$$

for all $\varphi \in V_{k}$ and all $\mu \in \Lambda_{k}$.

The consequences of this substitution are discussed in Remark 4.3. The basic idea for deriving the error estimation consists in the formulation of the auxiliary problem:

Auxiliary problem 4.2 Find $u_{\star}^{n} \in V_{k}$, such that the variational equation

$c\left(u_{\star}^{n}, \varphi\right)=\left(F_{h}^{n}, \varphi\right)-\left\langle\lambda_{H}^{n}, \gamma_{\mid \Gamma_{C}}(\varphi)\right\rangle$

holds for all $\varphi \in V_{k}$.

Auxiliary problem 4.2 corresponds to the first line of Auxiliary problem 4.1 , but with the discrete Lagrange multiplier $\lambda_{H}^{n}$ instead of $\tilde{\lambda}^{n}$. 
We observe that $u_{h}^{n}$ is also a discrete solution of Auxiliary problem 4.2. Therefore, the term $\left\|u_{\star}^{n}-u_{h}^{n}\right\|$ can be estimated by any error estimator $\eta_{\star}^{n}>0$ known for variational equations, i.e.,

$$
\left\|u_{\star}^{n}-u_{h}^{n}\right\|^{2} \leq C_{\star}\left(\eta_{\star}^{n}\right)^{2} .
$$

with a constant $C_{\star}>0$ independent of $V_{h}^{n}$ and $\Lambda_{H}^{n}$.

The derivation of the error estimator for the spatial error of the time-dependent Signorini problem can be sketched as follows: We show (see Prop. 4.1) that

$$
\left\|\tilde{u}^{n}-u_{h}^{n}\right\|^{2}+\left\|\tilde{\lambda}^{n}-\lambda_{H}^{n}\right\|^{2} \leq \hat{C}\left(\left\|u_{\star}^{n}-u_{h}^{n}\right\|^{2}+s_{0}^{2}+s_{1}^{2}\right)
$$

with a constant $\hat{C}>0$ independent of $h$ and $H$. Here, $\|\cdot\|$ denotes the norm correponding to the related function spaces. We use the $H^{1}(\Omega)$-norm for $V_{k}$ and the $\|\cdot\|_{-1 / 2, \Gamma_{C}}$-norm for functions in $H^{-1 / 2}\left(\Gamma_{C}\right)$. The additional terms $s_{0}$ and $s_{1}$ are defined in Remark 4.1 below. Then, an error estimation is obtained by

$\left\|\tilde{u}-u_{h}^{n}\right\|^{2}+\left\|\tilde{\lambda}^{n}-\lambda_{H}^{n}\right\|^{2} \leq C\left(\eta^{n}\right)^{2}$

with $C>0$ and an error estimator $\eta^{n}$ defined as

$\eta^{n}:=\eta_{\star}^{n}+s_{0}+s_{1}$.

Lemma 4.1 There are constants $C^{\prime}, C^{\prime \prime} \in \mathbb{R}_{>0}$, such that

$$
\begin{aligned}
& \left\|\tilde{u}^{n}-u_{h}^{n}\right\|^{2}+\left\|\tilde{\lambda}^{n}-\lambda_{H}^{n}\right\|^{2} \\
\leq & C^{\prime}\left\|u_{\star}^{n}-u_{h}^{n}\right\|^{2}+C^{\prime \prime}\left\langle\tilde{\lambda}^{n}-\lambda_{H}^{n}, \gamma_{\mid \Gamma_{C}}\left(u_{h}^{n}\right)-g^{n}\right\rangle .
\end{aligned}
$$

Proof. Let $v_{0}>0$ be the constant of continuity of $c$ and let $v_{1}$ be its constant of ellipticity. Then, we obtain by the ellipticity of $c$

$$
\begin{aligned}
& v_{1}\left\|\tilde{u}^{n}-u_{h}^{n}\right\|^{2} \\
\leq & c\left(\tilde{u}^{n}-u_{h}^{n}, \tilde{u}^{n}-u_{h}^{n}\right) \\
= & c\left(\tilde{u}^{n}-u_{\star}^{n}, \tilde{u}^{n}-u_{h}^{n}\right)+c\left(u_{\star}^{n}-u_{h}^{n}, \tilde{u}^{n}-u_{h}^{n}\right)
\end{aligned}
$$

Equation (4.1) and the continuity of $c$ lead to

$$
\begin{aligned}
& c\left(\tilde{u}^{n}-u_{\star}^{n}, \tilde{u}^{n}-u_{h}^{n}\right)+c\left(u_{\star}^{n}-u_{h}^{n}, \tilde{u}^{n}-u_{h}^{n}\right) \\
\leq & \left\langle\lambda_{H}^{n}-\tilde{\lambda}^{n}, \gamma_{\Gamma_{C}}\left(\tilde{u}^{n}-u_{h}^{n}\right)\right\rangle+v_{0}\left\|u_{\star}^{n}-u_{h}^{n}\right\|\left\|\tilde{u}^{n}-u_{h}^{n}\right\|
\end{aligned}
$$

Using (4.3) and Young's inequality, we get

$$
\begin{aligned}
& \left\langle\lambda_{H}^{n}-\tilde{\lambda}^{n}, \gamma_{\Gamma_{C}}\left(\tilde{u}^{n}-u_{h}^{n}\right)\right\rangle+v_{0}\left\|u_{\star}^{n}-u_{h}^{n}\right\|\left\|\tilde{u}^{n}-u_{h}^{n}\right\| \\
= & \left\langle\lambda_{H}^{n}-\tilde{\lambda}^{n}, \gamma_{\Gamma_{C}}\left(\tilde{u}^{n}\right)-g^{n}\right\rangle+\left\langle\tilde{\lambda}^{n}-\lambda_{H}^{n}, \gamma_{\Gamma_{C}}\left(u_{h}^{n}\right)-g^{n}\right\rangle \\
& +v_{0}\left\|u_{\star}^{n}-u_{h}^{n}\right\|\left\|\tilde{u}^{n}-u_{h}^{n}\right\| \\
\leq & \left\langle\tilde{\lambda}^{n}-\lambda_{H}^{n}, \gamma_{\Gamma_{C}}\left(u_{h}^{n}\right)-g^{n}\right\rangle+v_{0}\left\|u_{\star}^{n}-u_{h}^{n}\right\|\left\|\tilde{u}^{n}-u_{h}^{n}\right\| \\
\leq & \left\langle\tilde{\lambda}^{n}-\lambda_{H}^{n}, \gamma_{\Gamma_{C}}\left(u_{h}^{n}\right)-g^{n}\right\rangle+\varepsilon\left\|\tilde{u}^{n}-u_{h}^{n}\right\|^{2}+\frac{v_{0}^{2}}{4 \varepsilon}\left\|u_{\star}^{n}-u_{h}^{n}\right\|^{2} .
\end{aligned}
$$

Therefore, it holds

$$
\begin{aligned}
\left\|\tilde{u}^{n}-u_{h}^{n}\right\|^{2} \leq \frac{v_{0}^{2}}{4 \varepsilon\left(v_{1}-\varepsilon\right)}\left\|u_{\star}^{n}-u_{h}^{n}\right\|_{1}^{2} & \\
& \quad+\frac{1}{v_{1}-\varepsilon}\left\langle\lambda-\lambda_{H}^{n}, \gamma_{\Gamma_{C}}\left(u_{h}^{n}\right)-g^{n}\right\rangle
\end{aligned}
$$

with $0<\varepsilon<v_{1}$. In [43], it is proven that

$\left\|\tilde{\lambda}^{n}-\lambda_{H}^{n}\right\|^{2} \leq \tilde{C}\left\|\tilde{u}^{n}-u_{\star}^{n}\right\|^{2}$

with a constant $\tilde{C}>0$. This and inequality (4.4) yield

$$
\begin{aligned}
& \left\|\tilde{u}^{n}-u_{h}^{n}\right\|^{2}+\left\|\tilde{\lambda}^{n}-\lambda_{H}^{n}\right\|^{2} \\
\leq & \left\|\tilde{u}^{n}-u_{h}^{n}\right\|^{2}+\tilde{C}\left\|\tilde{u}^{n}-u_{\star}^{n}\right\|^{2} \\
\leq & \left\|\tilde{u}^{n}-u_{h}^{n}\right\|^{2}+\tilde{C}\left(\left\|\tilde{u}^{n}-u_{h}^{n}\right\|^{2}+\left\|u_{\star}^{n}-u_{h}^{n}\right\|^{2}\right) \\
\leq & C^{\prime}\left\|u_{\star}^{n}-u_{h}^{n}\right\|^{2}+C^{\prime \prime}\left\langle\lambda-\lambda_{H}^{n}, \gamma_{\Gamma_{C}}\left(u_{h}^{n}\right)-g^{n}\right\rangle
\end{aligned}
$$

with $C^{\prime}:=(1+\tilde{C}) /\left(4 \varepsilon\left(v_{1}-\varepsilon\right)\right)+\tilde{C}$ and $C^{\prime \prime}:=(1+\tilde{C}) /\left(v_{1}-\right.$ $\varepsilon)$.

As mentioned above, we are able to get rid of the term $\left\|u_{\star}^{n}-u_{h}^{n}\right\|$ by using an error estimator $\eta_{\star}^{n}$ for the auxiliary problem. Lemma 4.1 leads to

$$
\begin{aligned}
& \left\|\tilde{u}^{n}-u_{h}^{n}\right\|^{2}+\left\|\tilde{\lambda}^{n}-\lambda_{H}^{n}\right\|^{2} \\
\leq & C^{\prime} C_{\star}\left(\eta_{\star}^{n}\right)^{2}+C^{\prime \prime}\left\langle\lambda^{n}-\lambda_{H}^{n}, \gamma_{\Gamma_{C}}\left(u_{h}^{n}\right)-g^{n}\right\rangle .
\end{aligned}
$$

The remaining term $\left\langle\tilde{\lambda}^{n}-\lambda_{H}^{n}, \gamma_{\Gamma_{C}}\left(u_{h}^{n}\right)-g^{n}\right\rangle$ is estimated by

Lemma 4.2 Let $v_{0}>0$ be the constant of continuity of $c$. Furthermore, let

$d \in \tilde{K}^{n}:=\left\{v \in V_{k} \mid g^{n}-\gamma_{\Gamma_{C}}\left(u_{h}^{n}\right)-\gamma_{\mid \Gamma_{C}}(v) \leq 0\right\}$

and $\varepsilon>0$. Then, there holds

$$
\begin{gathered}
\left\langle\tilde{\lambda}^{n}-\lambda_{H}^{n}, \gamma_{\Gamma_{C}}\left(u_{h}^{n}\right)-g^{n}\right\rangle \\
\leq \frac{\varepsilon}{2}\left\|\tilde{u}^{n}-u_{h}^{n}\right\|^{2}+\frac{(1+\varepsilon) v_{0}^{2}}{2 \varepsilon}\|d\|^{2} \\
+\frac{1}{2}\left\|u_{\star}^{n}-u_{h}^{n}\right\|^{2}+\left|\left(\lambda_{H}^{n}, \gamma_{\Gamma_{C}}(d)\right)\right| .
\end{gathered}
$$

Proof. Inserting 0 and $2 \lambda_{H}^{n}$ in (3.8) yields

$$
\left\langle\lambda_{H}^{n}, \gamma_{\Gamma_{C}}\left(u_{h}^{n}\right)-g^{n}\right\rangle=0 .
$$

Furthermore, we get

$$
\begin{aligned}
& \left\langle\tilde{\lambda}^{n}, \gamma_{\Gamma_{C}}\left(u_{h}^{n}\right)-g^{n}\right\rangle \\
= & -\left\langle\tilde{\lambda}^{n}, g^{n}-\gamma_{\Gamma_{C}}\left(u_{h}^{n}\right)-\gamma_{\mid \Gamma_{C}}(d)\right\rangle-\left\langle\tilde{\lambda}^{n}, \gamma_{\Gamma_{C}}(d)\right\rangle \\
\leq & c\left(\tilde{u}^{n}, d\right)-\left(F_{h}^{n}, d\right),
\end{aligned}
$$

where the defintion of $\tilde{K}^{n}$ (4.5) and equation (4.1) have been used. The continuity of $c$ and Young's inequality lead to

$$
\begin{aligned}
& c\left(\tilde{u}^{n}, d\right)-\left(F_{h}^{n}, d\right) \\
= & c\left(\tilde{u}^{n}-u_{h}^{n}, d\right)+c\left(u_{h}^{n}, d\right)-\left(F_{h}^{n}, d\right) \\
\leq & v_{0}\left\|\tilde{u}^{n}-u_{h}^{n}\right\|\|d\|+c\left(u_{h}^{n}, d\right)-\left(F_{h}^{n}, d\right) \\
\leq & \frac{\varepsilon}{2}\left\|\tilde{u}^{n}-u_{h}^{n}\right\|^{2}+\frac{v_{0}^{2}}{2 \varepsilon}\|d\|^{2}+c\left(u_{h}^{n}, d\right)-\left(F_{h}^{n}, d\right) .
\end{aligned}
$$


The term $c\left(u_{h}^{n}, d\right)-\left(F_{h}^{n}, d\right)$ is estimated using equation (4.3), the continuity of $c$, and Young's inequality as follows:

$$
\begin{aligned}
& c\left(u_{h}^{n}, d\right)-\left(F_{h}^{n}, d\right) \\
= & c\left(u_{h}^{n}-u_{\star}^{n}, d\right)-\left(\lambda_{H}^{n}, \gamma_{\Gamma_{C}}(d)\right) \\
\leq & v_{0}\left\|u_{\star}^{n}-u_{h}^{n}\right\|\|d\|-\left(\lambda_{H}^{n}, \gamma_{\Gamma_{C}}(d)\right) \\
\leq & \frac{1}{2}\left\|u_{\star}^{n}-u_{h}^{n}\right\|^{2}+\frac{v_{0}^{2}}{2}\|d\|^{2}+\left|\left(\lambda_{H}^{n}, \gamma_{\Gamma_{C}}(d)\right)\right| .
\end{aligned}
$$

Eventually, we obtain an a posteriori error estimate by the following proposition:

Proposition 4.1 There exists a constant $C>0$ independent of $V_{h}^{n}$ and $\Lambda_{H}^{n}$, such that

$$
\begin{aligned}
& \left\|\tilde{u}^{n}-u_{h}^{n}\right\|^{2}+\left\|\tilde{\lambda}^{n}-\lambda_{H}^{n}\right\|^{2} \\
\leq & C\left(\left(\eta_{\star}^{n}\right)^{2}+\left\|\left(g^{n}-\gamma_{\Gamma_{C}}\left(u_{h}^{n}\right)\right)_{+}\right\|^{2}\right) \\
& +C\left|\left(\lambda_{H}^{n},\left(g^{n}-\gamma_{\Gamma_{C}}\left(u_{h}^{n}\right)\right)_{+}\right)\right|
\end{aligned}
$$

holds. Here, $f_{+}(x):=\max \{f(x), 0\}$ denotes the positive part of a function $f$.

Proof. Combining Lemma 4.1 and Lemma 4.2 yields

$$
\begin{aligned}
& \left\|\tilde{u}^{n}-u_{h}^{n}\right\|^{2}+\left\|\tilde{\lambda}^{n}-\lambda_{H}^{n}\right\|^{2} \\
\leq & C^{\prime} C_{\star}\left(\eta_{\star}^{n}\right)^{2}+C^{\prime \prime}\left\langle\tilde{\lambda}^{n}-\lambda_{H}^{n}, \gamma_{\Gamma_{C}}\left(u_{h}^{n}\right)-g^{n}\right\rangle \\
\leq & \left(C^{\prime}+\frac{1}{2} C^{\prime \prime}\right) C_{\star}\left(\eta_{\star}^{n}\right)^{2}+C^{\prime \prime} \frac{\varepsilon}{2}\left\|\tilde{u}^{n}-u_{h}^{n}\right\|^{2} \\
& +C^{\prime \prime}\left(\frac{(1+\varepsilon) v_{0}^{2}}{2 \varepsilon}\|d\|^{2}+\left|\left(\lambda_{H}^{n}, \gamma_{\Gamma_{C}}(d)\right)\right|\right) .
\end{aligned}
$$

Choosing $0<\varepsilon<2 / C^{\prime \prime}$, we get

$$
\begin{aligned}
& \left(1-\frac{C^{\prime \prime} \varepsilon}{2}\right)\left\|\tilde{u}^{n}-u_{h}^{n}\right\|^{2}+\left\|\tilde{\lambda}^{n}-\lambda_{H}^{n}\right\|^{2} \\
\leq & \tilde{C}\left(\left(\eta_{\star}\right)^{2}+\|d\|^{2}+\left|\left(\lambda_{H}^{n}, \gamma_{\mid \Gamma_{C}}(d)\right)\right|\right)
\end{aligned}
$$

with

$\tilde{C}:=\max \left\{\left(C^{\prime}+\frac{C^{\prime \prime}}{2}\right) C_{\star}, \frac{C^{\prime \prime}(1+\varepsilon) v_{0}^{2}}{2 \varepsilon}, C^{\prime \prime}\right\}$.

The function $d$ in Lemma 4.2 can be chosen as the harmonic continuation $\hat{d}^{n}$ of $\left(g^{n}-\gamma_{\Gamma_{C}}\left(u_{h}^{n}\right)\right)_{+}$, which is characterised by the minimisation problem

$$
\left\|\hat{d}^{n}\right\|_{1}^{2}=\inf _{v \in W^{n}}\|v\|_{1}^{2}
$$

with

$$
W^{n}:=\left\{v \in V^{n} \mid \gamma_{\Gamma_{C}}(v)=\left(g^{n}-\gamma_{\Gamma_{C}}\left(u_{h}^{n}\right)\right)_{+}\right\} .
$$

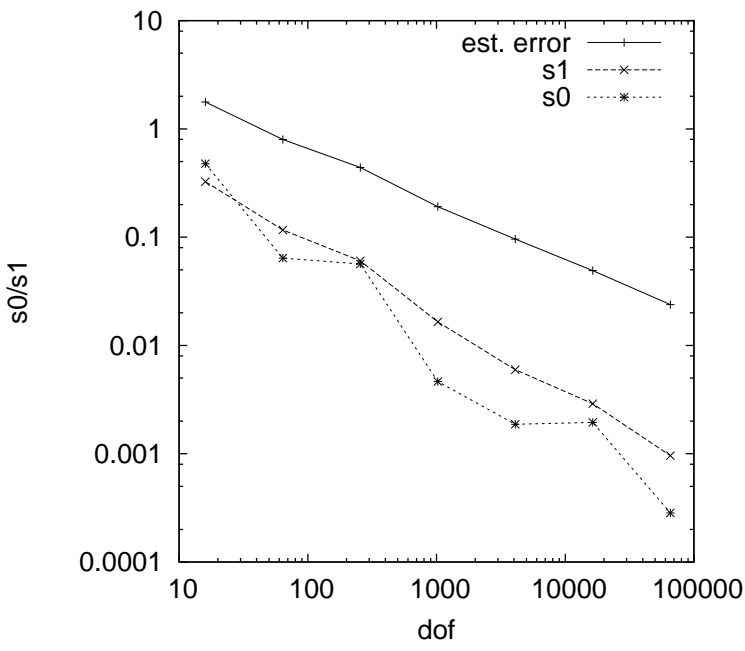

Fig. 4.1 Comparison of the values of the contributions to the presented error estimator.

For more details and alternatives in the choice of $d$ see [12]. Since there holds

$g^{n}-\gamma_{\Gamma_{C}}\left(u_{h}^{n}\right)-\gamma_{\Gamma_{C}}\left(\hat{d}^{n}\right) \leq 0$,

$\hat{d}^{n}$ is an element of $\tilde{K}^{n}$. From the definition of the norm $\|\cdot\|_{1 / 2, \Gamma_{C}}$, it follows

$$
\left\|\hat{d}^{n}\right\|_{1}=\left\|\left(g^{n}-\gamma_{\mid \Gamma_{C}}\left(u_{h}^{n}\right)\right)_{+}\right\|_{1 / 2, \Gamma_{C}} .
$$

Remark 4.1 All terms in the error estimate of Proposition 4.1 can be interpreted as typical sources of errors in contact problems. The term $s_{1}:=\left\|\left(g^{n}-\gamma_{\Gamma_{C}}\left(u_{h}^{n}\right)\right)_{+}\right\|$measures the error of the geometrical contact condition and the term $s_{0}:=\left|\left(\lambda_{H}^{n},\left(g^{n}-\gamma_{\mid \Gamma_{C}}\left(u_{h}^{n}\right)\right)_{+}\right)\right|$measures the violation of the complementarity condition.

Remark 4.2 In our numerical tests, the term $s_{1}$ always turned out to be smaller than the terms $s_{0}$ and $\eta^{n}$, see Figure 4.1. Since it is difficult to split this term into its elementwise contributions, it is neglected for the mesh refinement strategy.

In order to apply the error estimation of Proposition 4.1, we have to specify an appropriate error estimator $\eta_{\star}^{n}$ for Auxiliary problem 4.2. In principle, each error estimator known from literature of variational equations is possible to be used. See [46] or [47] for an overview. For the sake of completeness, a residual based error estimator for Auxiliary problem 4.2 is specified:

$$
\begin{aligned}
\left(\eta_{\star}^{n}\right)^{2} & :=\sum_{K \in \mathbb{T}^{n}} \eta_{K}^{2} \\
\eta_{K}^{2} & :=h_{K}^{2}\|r\|_{L^{2}(K)}^{2}+h_{K}\|R\|_{L^{2}(\partial K)}^{2}
\end{aligned}
$$


with

$r:=F_{h}^{n}+\frac{1}{2} k^{2} \triangle u_{h}^{n}-u_{h}^{n}$

$R:= \begin{cases}-\frac{1}{2} k^{2}\left(q-\frac{\partial u_{h}^{n}}{\partial v_{d}}\right) & \text { on } \partial \Omega \\ -\frac{1}{4} k^{2}\left[\frac{\partial u_{h}^{n}}{\partial v_{d}}\right] & \text { else. }\end{cases}$

The quantity $R$ represents the jump discontinuity in the approximation to the normal flux on the interface. We set $q=0$ on $\Gamma_{N}$ and $q=-\lambda_{H}^{n}$ on $\Gamma_{C}$. See [47], Section 2.2, for more details.

Remark 4.3 We have used the discrete value $F_{h}^{n}$ instead of $F^{n}$ in Auxiliary problem 4.1, i.e., Proposition 4.1 specifies a temporal local error estimator for the spatial discretisation error. This technique is commonly used in the derivation of error estimators for numerical methods for ordinary differential equations, see, e.g., [48]. The presented error estimator expresses the spatial error distribution in the single time steps. But it only provides information about the global error under the assumption $F_{h}^{n} \approx F^{n}$, which should hold for small $k$ and $h$. An a priori error analysis of the Newmark method in the context of dynamic contact problems is needed to make a precise statement. To the best of the authors' knowledge, such an analysis does not exist and cannot be derived by standard techniques due to the low regularity of the continuous solution.

Remark 4.4 The presented error estimate is not restricted to the Newmark method. It can easily be used for other similar time stepping schemes. It was tested by the authors for the Generalized- $\alpha$ method, the application of which to dynamic contact problems is presented in $[34,35]$.

\section{Adaptive Algorithm}

In general, adaptive algorithms for dynamic problems are based on refinement strategies, which are known from static problems, see, e.g., $[46,49]$ for a survey of adaptive algorithms for static problems. Commonly used adaptive algorithms for time dependent problems, see, e.g., [3,50], perform an adaptive refinement process using a prescribed tolerance in every time step. This refinement process is independent of previous and subsequent time steps. The crucial point is that the time interval is passed only once. The tolerance limit cannot be reached, if the solution in the previous time step has not been calculated exactly enough. Moreover, the difference of the meshes of two succsessive time steps may significantly increase the error. Usually, rapid changes of the problem parameters are the reason for this behaviour.

In dynamic Signorini problems, the problem parameters change rapidly and, thus, the above mentioned algorithms are not appropriate. An alternative is given by algorithms based on the ideas in $[51,52]$. The adaptive solution algorithm is presented in Algorithm 5.1. It is split into several cycles. The whole time interval is passed in every cycle. First, the approximative solution of the whole problem is determined and the error is estimated. Then, the mesh is refined via an appropriate spatial refinement strategy, which can be chosen by the user, e.g., a fixed fraction strategy, see $[46,49]$. Multiple hanging nodes in space and time may be generated by this refinement process. This may cause severe problems w.r.t. energy conservation. Therefore, the multiple hanging nodes in space are immediately removed by a spatial regularisation algorithm. After finishing the whole time interval the multiple hanging nodes in time are removed, which closely connects the meshes of different time steps. Then, the stopping criterion is checked. Possible stopping criterions are: The number of degrees of freedom is greater than a specified number, the number of cycles is greater than a given number or the estimated error is smaller than a specified tolerance. A more detailed presentation of this adaptive algorithm, its extensions and the mathematical analysis will be given in a separate article.

Algorithm 5.1 Adaptive solving algorithm

1. Set $l=1$.

2. Set $n=1$ and determine inital values $u_{h_{l}}^{0}, v_{h_{l}}^{0}, u_{h_{l}}^{0} \in V_{h_{l}}^{1}$.

3. Determine $u_{h_{l}}^{n}, v_{h_{l}}^{n}, u_{h_{l}}^{n}, \lambda_{H_{l}}^{n}$ based on $(3.1,3.2,3.7,3.8)$.

4. Evaluate the error estimator $\eta_{l}^{n}$.

5. Set $\mathbb{T}_{l+1}^{n}=\mathbb{T}_{l}^{n}$ and refine $\mathbb{T}_{l+1}^{n}$ according to the chosen refinement strategy based on $\eta_{l}^{n}$.

6. Remove all multiple hanging nodes in space with Algorithm 5.2.

7. If $n<N$ set $n \leftarrow n+1$ and go to 3

8. Remove all double hanging nodes in time with Algorithm 5.3 .

9. If the stopping criterion is fulfilled, then stop, else set $l \leftarrow l+1$ and go to 2 .

\section{Algorithm 5.2 Spatial regularisation algorithm}

1. Mark all cells with multiple hanging nodes.

2. If no cells are marked, then stop.

3. Refine all marked cells.

4. Go to 1

\section{Algorithm 5.3 Temporal regularisation algorithm}

1. $\operatorname{Set} n=2$.

2. Mark all cells with multiple backward hanging nodes in time in $\mathbb{T}^{n}$.

3. Refine all marked cells in $\mathbb{T}^{n}$.

4. If $n<N$, set $n \leftarrow n+1$ and go to 2 . 


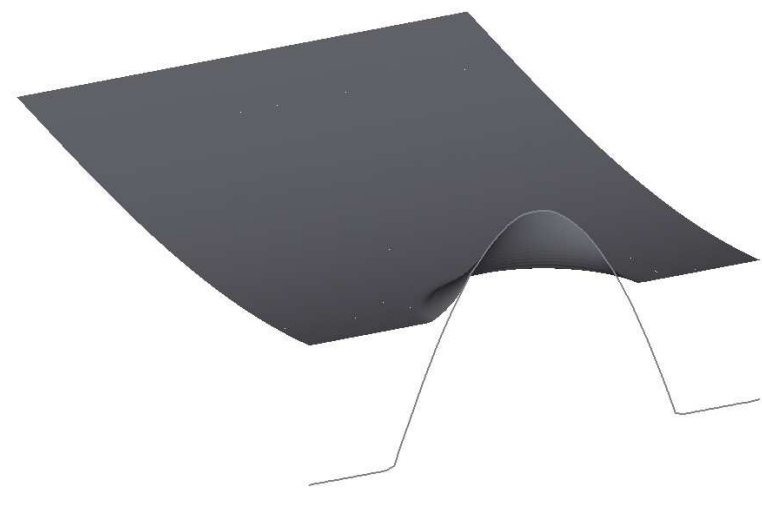

Fig. 6.1 Geometry of the simplified Signorini example

5. Set $n=N-1$.

6. Mark all cells with multiple forward hanging nodes in time in $\mathbb{T}^{n}$.

7. Refine all marked cells in $\mathbb{T}^{n}$.

8. If $n>1$, set $n \leftarrow n-1$ and go to 6 .

9. Stop.

\section{Numerical Results}

The error estimator and the adaptive algorithm are tested for two examples. The first one is a simplified Signorini problem and the second one is a full 2D Signorini problem.

\subsection{A simplified Signorini example}

We set $\Omega:=[0,1]^{2}$ and $I:=[0,1]$. The initial values are $u_{0}\left(x_{1}, x_{2}\right):=0$ and $v_{0}\left(x_{1}, x_{2}\right):=-\sin \left(\frac{1}{2} \pi x_{1}\right)$. Furthermore, we set $\Gamma_{D}:=\left\{x \in \Omega \mid x_{1}=0\right\}, \Gamma_{C}:=\left\{x \in \Omega \mid x_{1}=1\right\}$ and $\Gamma_{N}:=\partial \Omega \backslash\left(\Gamma_{C} \cap \Gamma_{D}\right)$. The rigid foundation is

$g:=\sin \left(\pi x_{2}\right)-1.05$

The length of the time steps $k$ is chosen as 0.0025 . The initial mesh size $h_{0}$ is 0.0625 . Five refinement cycles are performed, whereas a fixed fraction strategy with constant refinement fraction of $50 \%$ and no coarsening is used.

The geometry of the presented problem is illustrated in Figure 6.1. Meshes for different time steps are presented in Figure 6.2, the corresponding movie is shown in Animation 1. The mesh in Figure 6.2 (a) corresponds to the time step, immediately before the first contact between the membrane and the rigid foundation takes place. In Figure 6.2 (b)-(i), the membrane gets into contact with the obstacle and the contact zone is adaptively refined. We observe a moving front,

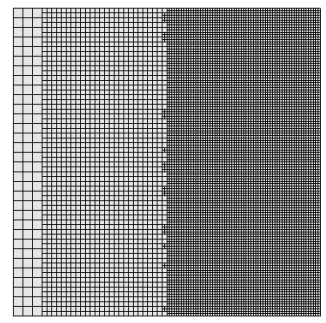

(a) Mesh at $n=20$

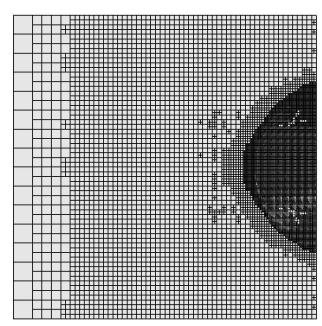

(c) Mesh at $n=100$

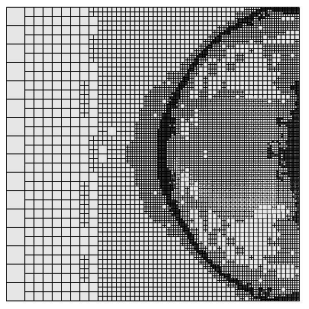

(e) Mesh at $n=200$

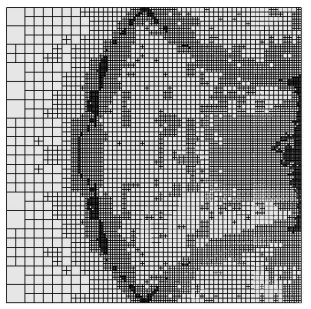

(g) Mesh at $n=300$

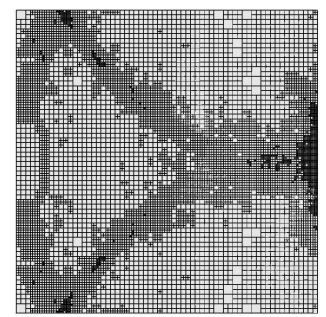

(i) Mesh at $n=400$

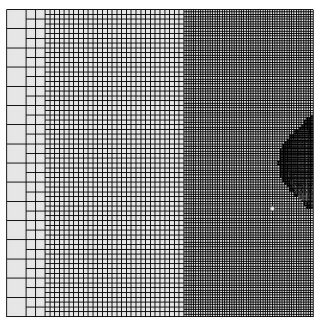

(b) Mesh at $n=50$

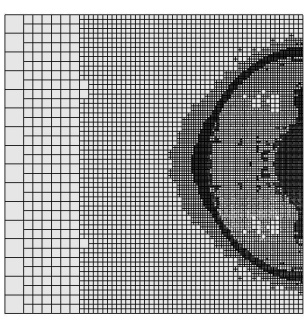

(d) Mesh at $n=150$

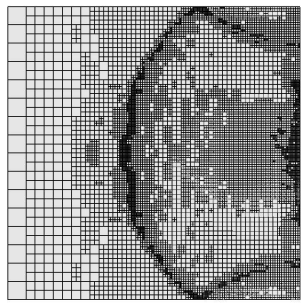

(f) Mesh at $n=250$

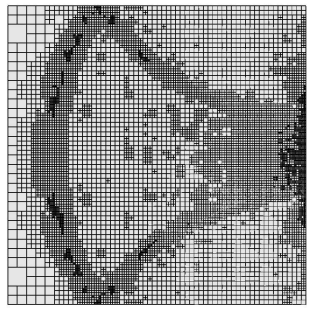

(h) Mesh at $n=350$
Fig. 6.2 Meshes for different time steps of the simplified Signorini example 


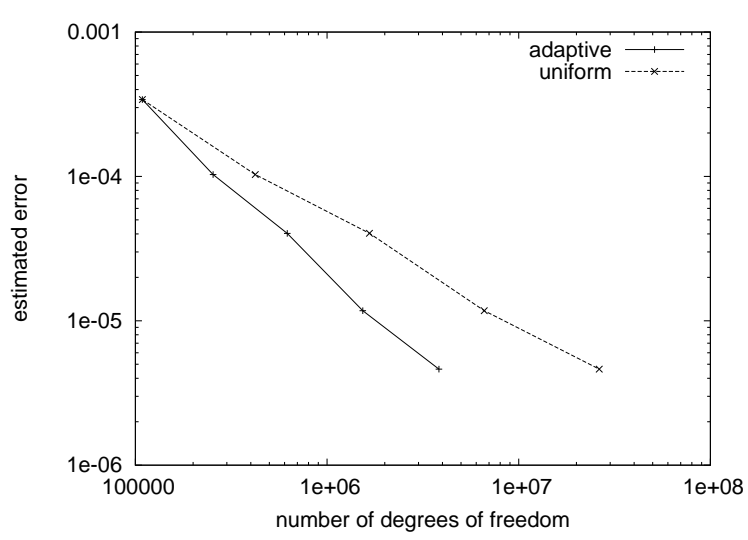

Fig. 6.3 Estimated error for adaptive and uniform refinement in the simplified Signorini example

which is resolved by the adaptive meshes. In Figure 6.3 the estimated convergence for adaptive and uniform refinement is compared. The estimated error is measured by

$\eta=\max _{1 \leq n \leq N} \eta^{n}$

where $\eta^{n}$ is given in Proposition 4.1. The number of degrees of freedom is the sum of the number of degrees of freedom of all single time steps. It is obvious, that the adaptive refinement is more efficient than the uniform refinement. One achieves the same accuracy with nearly a factor of 10 less unknowns.

\subsection{A Signorini example}

Here, a bar of length $0.2 \mathrm{~m}$ and height $0.05 \mathrm{~m}$ is considered. The domain is $\Omega:=[0,0.2] \times[0,0.05]$ and the time interval is $I:=\left[0,2.5 \cdot 10^{-3}\right]$. The bar is modelled using a linear elastic material law in a plain strain situation with $E:=$ $73 \cdot 10^{9} \mathrm{MPa}$ and $\bar{v}:=0.33$. The density is $\rho:=2770 \mathrm{~kg} / \mathrm{m}^{2}$. The bar is fixed at the left boundary $\Gamma_{D}=\left\{x \in \Omega \mid x_{1}=0\right\}$. The possible contact surface is given by the set

$\Gamma_{C}=\left\{x \in \Omega \mid x_{1} \geq 0.15 \wedge x_{2}=0\right\}$.

There are nonhomogeneous Neumann boundary conditions on $\Gamma_{N}=\left\{x \in \Omega \mid x_{1} \geq 0.1 \wedge x_{2}=0.05\right\}$ with

$q:=3.75 \cdot 10^{7} \mathrm{~N} / \mathrm{m}^{2}$.

The rigid foundation is given by the set

$\left\{x \in \mathbb{R}^{2} \mid 0 \leq x_{1} \leq 0.2 \wedge x_{2} \leq-0.005\right\}$.

The length of the time steps is $10^{-5}$ and the initial mesh size $h_{0}$ is $6.25 \cdot 10^{-3}$. Again, five refinement cycles are performed, whereas a fixed fraction strategy with constant refinement fraction of $50 \%$ without coarsening is used.

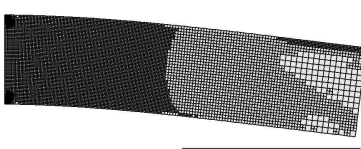

(a) Mesh at $n=20$

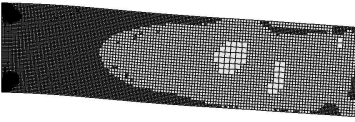

(c) Mesh at $n=50$

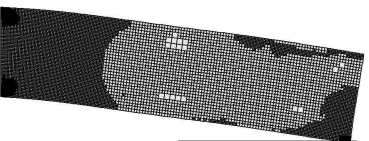

(e) Mesh at $n=90$

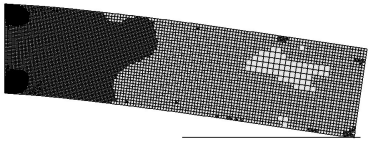

(g) Mesh at $n=211$

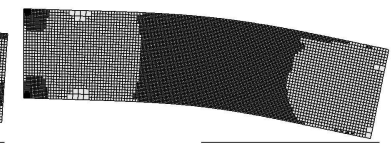

(b) Mesh at $n=25$

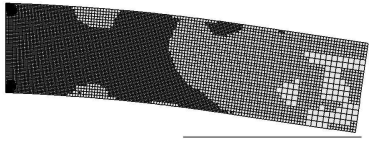

(f) Mesh at $n=100$

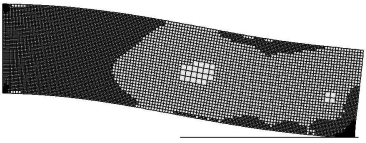

(h) Mesh at $n=215$

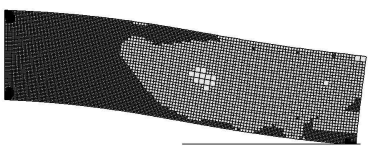

(i) Mesh at $n=217$

Fig. 6.4 Meshes for different time steps of the 2D Signorini example

In Figure 6.4 meshes for different time steps are presented the corresponding movie is contained in Animation 2. The displacement is scaled by a factor of 5. During the calculation, contact between the bar and the rigid foundation occurs several times. In the Figures 6.4 (a)-(c) and (d)(f) a sequence is depicted, which starts before contact takes places and ends after contact. The influence of contact to the 


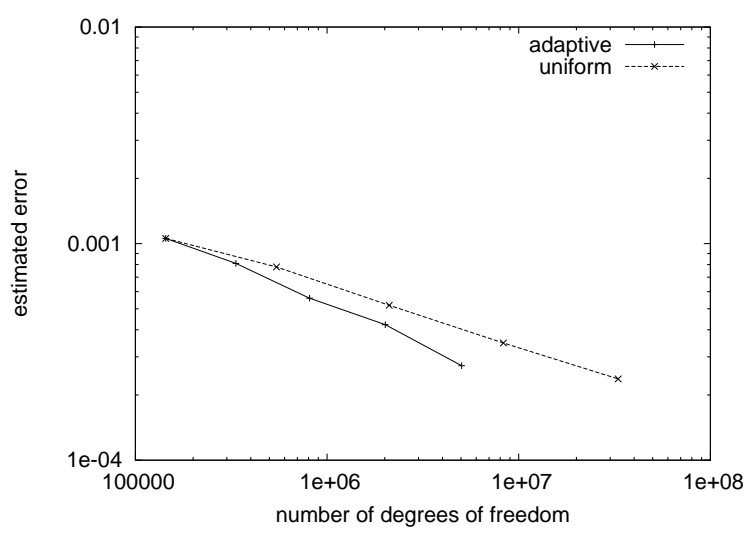

Fig. 6.5 Estimated error for adaptive and uniform refinement in the Signorini example

mesh is obvious. The last sequence (g)-(i) shows the change of the mesh during contact. The refined zone and the contact zone grow and shrink simultanously. The performance of the adaptive refinement is compared with the uniform refinement in Figure 6.5, where the same variables are used as in Figure 6.3. As in the example above, the application of the adaptive method is more efficient.

\section{Conclusions}

The presented space adaptive scheme for dynamic Signorini problems shows a significant improvement. More sophisticated refinement strategies can probably further enhance the efficiency. However, not every strategy known from contact free problems seems to be suited for adaptive schemes for contact problems. E.g., the refinement strategy presented in [52] compares the refinement indicators over all time steps. The method has been tested by the authors, but the results are not satisfactory. The contact zone is not resolved, before the first contact takes place. Thus, the algorithm is not able to detect the moment of the first contact exactly, so that the error increases significantly.

Another method to improve the discretisation is given by time adaptivity; error estimators for the Newmark method as presented in [6] can be used. This technique will be considered in future works.

The difficulties discussed in Remark 4.3 and the separation of the spatial and temporal discretisation complicate the derivation of rigorous a posteriori error estimators. A way out could be the application of a space-time Galerkin method [53] and of DWR techniques [49].

\section{Acknowledgments}

This research work was supported by the Deutsche Forschungsgemeinschaft (DFG) within the Priority Program
1180, Prediction and Manipulation of Interaction between Structure and Process, and within the Collaborative Research Center 708, 3D-Surface Engineering of Tools for Sheet Metal Forming - Manufacturing, Modelling, Machining.

\section{References}

1. Weinert K, Blum H, Jansen T, Rademacher A (2007) Simulation based optimization of the NC-shape grinding process with toroid grinding wheels. Prod Eng 1(3):245-252

2. Johnson C (1993) Discontinuous Galerkin Finite Element Methods for Second Order Hyperbolic Problems. Comput Methods Appl Mech Eng 107:117-129

3. Li X, Wiberg N-E (1998) Implementation and Adaptivity of a Space-Time Finite Element Method for Structural Dynamics. Comput Methods Appl Mech Eng 156:211-229

4. Aubry D, Lucas D, Tie B (1999) Adaptive Strategy for Transient/Coupled Problems. Applications to Thermoelasticity and Elastodynamics. Comput Methods Appl Mech Eng 176:41-50

5. Bangerth W, Rannacher R (1999) Finite Element Approximation of the Acoustic Wave Equation: Error Control and Mesh Adaptation. East-West J Numer Math 7(4):263-282

6. Xie Y M, Zienkiewicz O C (1991) A Simple Error Estimator and Adaptive Time Stepping Procedure for Dynamic Analysis. Earth Eng Struct Dyn 20:871-887

7. Li X, Wiberg N-E, Zeng L F (1992) A Posteriori Local Error Estimation and Adaptive Time-Stepping for Newmark Integration in Dynamic Analysis. Earth Eng Struct Dyn 21:555-571

8. Bornemann F A, Schemann M (1998) An Adaptive Rothe Method for the Wave Equation. Comput Visual Sci 1:137-144

9. Adjerid S (2002) A Posteriori Finite Element Error Estimation for Second-Order Hyperbolic Problems. Comput Methods Appl Mech Eng 191:4699-4719

10. Bernadi C, Süli E (2005) Time and Space Adaptivity for the Second-Order Wave Equation. Math Models Methods Appl Sci 15(2):199-225

11. Braess D (2005) A posteriori error estimators for obstacle problems - another look. Numer Math 101(3):415-421

12. Schröder A (2006) Fehlerkontrollierte adaptive $h$ - und $h p$-FiniteElemente-Methoden für Kontaktprobleme mit Anwendungen in der Fertigungstechnik. Bayreuther Math Schr 78 available also via http://hdl.handle.net/2003/22487

13. Johnson C (1992) Adaptive Finite Element Methods for the Obstacle Problem. Math Models Methods Appl Sci 2:483-487

14. Ainsworth M, Oden J T, Lee C Y (1993) Local A Posteriori Error Estimators for Variational Inequalities. Numer Methods Partial Differ Equ 9:23-33

15. Hoppe R H W, Kornhuber R (1994) Adaptive Multilevel Methods for Obstacle Problems SIAM J Numer Anal 31:301-323

16. Chen Z, Nochetto R (2000) Residual Type A Posteriori Error Estimates for Elliptic Obstacle Problems. Numer Math 84:527-548

17. Veeser A (2001) Efficient and Reliable A Posteriori Error Estimators for Elliptic Obstacle Problems. SIAM J Numer Anal 39:146-167

18. Nochetto R H, Siebert K G, Veeser A (2003) Pointwise A Posteriori Error Control for Elliptic Obstacle Problems. Numer Math 95:163-195

19. Bartels S, Carstensen C (2004) Averaging Techniques yield Reliable A Posteriori Finite Element Error Control for Obstacle Problems. Numer Math 99:225-249

20. Suttmeier F T (2005) On a Direct Approach to Adaptive FEDiscretisations for Elliptic Variational Inequalities. J Numer Math 13:73-80

21. Wohlmuth B I (2007) An A Posteriori Error Estimator for TwoBody Contact Problems on Non-Matching Meshes. J Sci Comput 33:25-45 
22. Braess D, Carstensen C, Hoppe R H W (2007) Convergence Analysis of a Conforming Adaptive Finite Element Method for an Obstacle Problem. Numer Math 107:455-471

23. Wriggers P, Vu Van T, Stein E (1990) Finite Element Formulation of Large Deformation Impact-Contact Problems with Friction. Comput Struct 37(3):319-331

24. Bathe K J, Chaudhary A B (1986) A Solution Method for Static and Dynamic Analysis of Three-Dimensional Contact Problems with Friction. Comput Struct 24(6):855-873

25. McDevitt T W, Laursen T A (2000) A Mortar-Finite Element Formulation for Frictional Contact Problems. Int J Numer Methods Eng 48(10): $1525-1547$

26. Puso M, Laursen T A (2002) A 3D Contact Smoothing Method using Gregory Patches. Int J Numer Methods Eng 54(8):1161-1194

27. Hager C, Hüeber S, Wohlmuth B I (2008) A Stable Energy Conserving Approach for Frictional Contact Problems based on Quadrature Formulas.Int J Numer Methods Eng 73:205-225

28. Deuflhard P, Krause R, Ertel S (2007) A contact-stabilized Newmark method for dynamical contact problems. Int J Numer Methods Eng 73(9): 1274-1290

29. Armero F, Petócz E (1998) Formulation and analysis of conserving algorithms for frictionless dynamic contact/impact problems. Comput Methods Appl Mech Eng 158:269-300

30. Laursen T A, Chawla V (1997) Design of Energy Conserving Algorithms for Frictionless Dynamic Contact Problems. Int J Numer Methods Eng 40:863-886

31. Kane C, Repetto E A, Ortiz M, Marsden J E (1999) Finite Element Analysis of nonsmooth contact. Comput Methods Appl Mech Eng 180:1-26

32. Pandolfi A, Kane C, Marsden J E, Ortiz M (2002) Timediscretized variational formulation of non-smooth frictional contact. Int J Numer Methods Eng 53:1801-1829

33. Talaslidis D, Panagiotopoulos P D (1982) A Linear Finite Element Approach to the Solution of the Variational Inequalities arising in Contact Problems of Structural Dynamics. Int J Numer Methods Eng 18:1505-1520

34. Czekanski A, Meguid S A (2001) Analysis of Dynamic Frictional Contact Problems using Variational Inequalities. Finite Elem Anal Des 37:6861-879

35. Rademacher A (2005) Finite-Elemente-Diskretisierungen für dynamische Probleme mit Kontakt. Diploma-Thesis, University of Dortmund. Available via http://hdl.handle.net/2003/22996

36. Laursen T A (2002) Computational Contact and Impact Mechanics. Springer, Berlin

37. Wriggers P (2002) Computational Contact Mechanics. John Wiley \& Sons Ltd, Chichester

38. Kikuchi N, Oden J T (1988) Contact Problems in Elasticity: A Study of Variational Inequalities and Finite Element Methods. SIAM, Studies in Applied Mathematics,

39. Evans L C (1998) Partial Differential Equations, American Mathematical Society, Providence

40. Panagiotopoulos P D (1985) Inequality Problems in Mechanics and Applications, Convex and Nonconvex Energy Functions. Birkhäuser, Basel

41. Newmark N M (1959) A Method of Computation for Structural Dynamics. Journal of the Engineering Mechanics Division ASCE 85(EM3):67-94

42. Hughes T J R (2000) The Finite Element Method. Dover Publications, Inc, Mineola

43. Schröder A (2009) Error control in $h$ - and $h p$-adaptive FEM for Signorini's problem. Institute for Mathematics, Humboldt Universität zu Berlin (ISSN 0863-0976), Preprint 09-05. Available via www.mathematik.hu-berlin.de/publ/pre/2009/p-list-09.html

44. Cea J (1978) Lectures on optimization - theory and algorithms. Springer, Berlin

45. Ekeland I, Temam R (1976) Convex analysis and variational problems, Studies in Mathematics and its Applications. North-Holland Publishing Company, Amsterdam
46. Verfürth R (1996) A Review of A Posteriori Error Estimation and Adaptive Mesh-Refinement Techniques, Wiley and Teubner, Chichester

47. Ainsworth M, Oden J T (2000) A Posteriori Error Estimation in Finite Element Analysis. John Wiley \& Sons, Inc, New York

48. Hermann M (2004) Numerik gewöhnlicher Differentialgleichungen. Oldenbourg Verlag, München

49. Bangerth W, Rannacher R (2003) Adaptive Finite Element Methods for Differential Equations. Birkhäuser, Basel

50. Schmidt A, Siebert K G (2004) Design of Adaptive Finite Element Software. Lecture Notes in Computational Science and Engineering, Springer, Berlin

51. Bangerth W, Rannacher R (2001) Adaptive Finite Element Techniques for the Acoustic Wave Equation. J Comp Acoustics 9(2):575591

52. Schmich M, Vexler B (2008) Adaptivity with dynamic meshes for space-time finite element discretizations of parabolic equations. SIAM J Sci Comput 30(1):369-393

53. Blum H, Jansen T, Rademacher A, Weinert K (2008) Finite Elements in Space and Time for Dynamic Contact Problems. Int J Numer Meth Eng 76:1632-1644 